changes in the lower extremities, of the type of so-called "varicose ulcers," "varicose eczema," and "varicose pigmentation," associated or not with pronounced varicose veins.

REFERENCES.

R. Virchow : Ueber die Chlorose und Anomalien im Gefässapparate, 1872. 0 . Rosenbach : Corset und Bleichsucht, Stuttgart, 189. Roch and G. Bickel: Bull. Soc. Méd. des Hôpitaux do Paris, 1924, erles iil, xlviii, p. 76. 'T. Deneke : Deut. med. Woch., 1924, 1, p. 902 but but suggested that it might be due to lessened consumption of alcoho Dermatologie et de Syphiligraphie, Paris, 1925, Series vi, vol. vi, pp. 1-18. This paper contains many references to previous English and French literature on the etiology of erythema induratum. 'Cf. Radcliffe Crocker' Diseases of the Skin, according to which Bazin himself did not describ lceration as a feature of his erythema induratum. ' $\mathbf{S}$. E. Dore an A. W. Stctt: Proc. Roy. Soc. Med., Dermat. Section, 1922, xv, p. 43. H. MacCormac : Ibid., 1923, xvi, p. 11. G. N. Meachen : Reported in Brit. Journ. Dermat., 1914, xxvi, p. 202. 10 S. N. Vendel : Ugeskrift for Joeger, Copenhagen, 1924, Ixxxvi, p.

\section{THE TREATMENT OF HAY FEVER.}

\section{FRANK COKE, F.R.C.S.}

Is popular parlance the term " hay fever" is used very loosely to include all forms of paroxysmal sneezing. In this article hay fever refers solely to the affection due specifically to sensitization to the pollens. The majority of cases are due to the grass pollens, and recur with great regularity from the end of May to the middle of July. A few cases, due to sensitization to the tree pollens, commence in April or the beginning of May. The identity of these should be discovered by means of the dermal tests before the season commences.

Sweet-smelling flowers and those which are highly coloured have heavy sticky pollens and are fertilized by insects; their pollens are not air-borne, and so have little effect in causing hay fever. The scent of these flowers will of ten provoke paroxysms in hay fever patients, but this can be disregarded in planning the treatment, as when desensitized to the grass pollens the patient's sensitiveness to the smell of flowers will disappear.

\section{Preventive Treatment.}

Sea voyages are effective, but sending patients to the seaside is of no use unless a guarantee can be given that the wind there will be from the sea. Avoidance of the pollen being, then, impossible, one of the following methods must be used to desensitize the patient.

(a) Desensitization of the Patient by Small Doses of the Pollen.-Judging by experience in desensitizing horse asthmatics, far greater trouble should be taken in using this method than is commonly observed. It would seem that we have to use up the patient's specific adzyme, as [ have called it, by means of minute doses of the protein spread over a period of many months without at any time giving a large enough dose to excite the formation of more specific adzyme. The patient is tested with dilutions of the protein, and a dilution ten times weaker than that which gives an appreciable reaction on the skin is used. Starting with a dose of 2 minims of this, weekly or bi-weekly doses are given, increasing each dose by 1 minim until the next dilution containing ten times more pollen is reached. We do the same with this, gradually increasing the dose until a higher dilution is required. All this must be done very slowly, making frequent dermal reactions to see how the course of treatment is progressing. When the stronger solutions are used the doses should be increased very slowly indeed. It is essential that the dilutions used to treat the patient are actually those with which he has been tested; otherwise they are bound to vary in strengthfirst, because the grass flowers from which the dilutions are made, though duly weighed, may be wind-blown and so carry varying amounts of pollens; and secondly, because the strength may deteriorate considerably after it is made. The method of giving a few doses shortly before the hay fever season, and especially daily doses, may appreciably lessen the patient's hay fever, but for another reason: anti-anaphylaxis takes place. However successful this may be it fails, because it gives no vrotection for the next season. Before we can say that the patient is cured we must not only obtain freedom from hay fever but from treatment as well.

(b) Desensitization by Non-specific Methods.-Probably any protein would effect this purpose, but the danger of the patient being sensitive to them is so great, especially in the case of horse serum, as to preclude their routine use. In my experience the " mixed coliform vaccine" originally suggested by Danysz is the most effective. Though an autogenous vaccine is undoubtedly preferable, heterogeneous mixtures may be used. These should contain some 40 per cent. of Bacillus coli, 40 per cent. of coliform variants, and 20 per cent. of intestinal streptococci. Doses varying from and rising from 20 to 1,000 million of these organisms are injected hypodermically, once or twice a week according to the nearness of the hay fever season. I have had several patients who have had a course of this treatment four years ago and who have passed through subsequent seasons without hay fever and also without the inconvenience of the yearly course of treatment. Treatment with this vaccine should be commenced in April and continued well into the hay fever season. But it is almost equally effective when given later in the season. It will often stop hay fever even when violently active, though the permanent desensitizing power is best conferred by the early course.

These two methods should not be used together, for the pollen injections will defeat the objects of the mixed coliform vaccine.

(c) Medicines are of very little use in controlling hay fever. The calcium salts are those most favoured. 1 find great value in the use of collosol calcium, which should be given when the hay fever season is due to commence in $1 / 2$ to $1 \mathrm{c.cm}$. doses intravenously.

(d) Cauterization of the nose is very useful for two purposes. If on examination of the nose before the hay fever season commences we find that the turbinals are covered with boggy swollen mucous membrane, cauterization should be undertaken to reduce them by scarring. The other use of the cautery is to touch the mucous membrane lightly and destroy some of the nerve endings and so lessen the extreme sensitiveness present. This should be done over the turbinals on either side, and particularly on the septum anterior to the bony outlet of the nose. With a blunt probe other tender spots can be searched throughout the whole area. I am in the habit of doing this cauterization about the middle of May, thus allowing just sufficient time for healing before the season commences.

\section{Symptomatic Treatment.}

(a) The patient should be placed in a cool dark room. The admittance of fresh air from the outside should be limited to the minimum, for the whole air is heavily laden with pollen. For the same reason the windows should be kept closed at night unless the warmth of the weather makes this impossible.

(b) The effect of the direct sunlight is so provocative of sneezing that dark glasses should be worn if the patient does not stay indoors. These should fit closely rouid the eyes, after the manner of motor goggles. Broad-rimmed hats and caps are often helpful.

(c) The patient is greatly assisted by taking steps to prevent the pollen passing on to the mucous membrane of the nose. Unfortunately, the worse the patient's hay fever is the more difficult it is to touch the nose without causing a paroxysm of sneezing. Simplest, perhaps, is a tube of vaseline with a long nozzle out of which sufficient can be squeezed just inside the nose. This can be pushed further up the nose by pinching that organ with the finger and thumb, assisted by sniffing it up. Liquid paraffin or olive oil may be used very frequently in a coarse sprav.

(d) Various medicaments may be added to the paints and sprays to meet the symptoms. Adrenaline and menthol will constrict the blood vessels and so lessen the swelling of the mucous membrane, allowing the orifices of the various sinuses to regain their patency. Cocaine and its surstitutes reduce the intense irritation of the nerve endings in the nose, and constrict the blood vessels, especially cocaine. Oily and greasy rehicles for these drugs soothe the mucous membrane and prevent fresh pollen getting to it. 
(e) Similar drugs may be used for the eye symptoms. In educated people, who can be trusted not to use the drugs to excess, the instillation of a few drops of cocaine (4 grains) and adrenaline (3 drachms to the ounce) will not only help the eyes, but, by passing down the lacrymal duct, will also allay the trouble in the nose. Used in an atomizer these may be sprayed up the nose and also into the eyes. In prescribing these drugs some estimate must be made of how often the patient will use them and, more particularly still, the kind of spray he will use them in, likewise the viscosity of the ingredients of the solution used.

(f) A search should be made for any microbic invaders which are adding to the patient's discomfort.

(g) Considerable comfort is experienced by dipping the whole face into a basin of cool water at bedtime. If sufficient salt is added the eyes may bo opened and washed in this. Keeping the lips and glottis closed some patients manage, by expanding the buccal cavity, to draw the solution into the nose and wash that at the same time. Such a washing often allows the patient to fall asleep with ease and comfort.

By these various methods much more can be done for the unfortunate sufferer from hay fever than was possible formerly.

\section{RECTAL INJECTION OF ETHER IN WHOOPING-COUGH. BY}

\section{ELGOOD, B.M.OxFond}

HOUSE-PHYSICIAN TO THE EVELINA HOSPITAL FOR SICK CHILDREN, SOUTHWARK.

MaNy methods are used in the treatment of whooping-cough; among the many drugs belladonna, opium, and quinine seem to be the favourites. Recently $x$-ray treatment has been suggested in America ${ }^{1}$ and in England. ${ }^{2}$. Vaccine treatment has also been tried on many cases. ${ }^{3}$ Ether was first suggested in 1914 by Audrain, whose results were published in 1920.* $\mathrm{He}$ used 1 to $2 \mathrm{c.cm}$. injected intramuscularly three times a day. Since then several other workers have published their results; Panayotatou stated that the duration of the disease could be reduced to twelve days after starting treatment by a dose of 1.5 to $3 \mathrm{c.cm}$. of ether given intragluteally on alternate days. ${ }^{5}$ Mason in America, who treated 26 cases in the same way, ${ }^{6}$ had 60 per cent. cured and 24 per cent. definitely improved. He also tried ether by the rectum on four cases, using $6 \mathrm{c.cm}$. of a 40 per cent. solution of ether in olive oil. Half of these were successful and half failures.

$\mathrm{Smith}^{7}$ has recently reviewed all the literature published since the war on the treatment of whooping-cough, but does not mention the rectal method of administering ether. It would seem that this is the first time that a large number of cases so treated has been put on record.

\section{Modi of Administration of Ermer.}

It has been the custom to administer the ether by subcutaneous or intramuscular injection. This is undoubtedly the most rapid method, but it is very unsatisfactory for children between the ages of 2 and 12. An enema by the rectum is much more suitable. The enema is painless-only two cases in this series complained of abdominal pain, rapidly passing off, and the majority of children raise no objection to the repetition of the treatment. The only diffioulty lies in getting a certain number of cases to retain it.

When ether was given by injection $I$ always used the intragluteal route. The dose was 5 to 10 minims of the ordinary anaesthetic ether. For the administration of an enema I used a full-sized male rubber catheter, attached by a small glass tube to a larger-bored rubber tube with a funnel at the other end. The ether was mixed with an equal amount of olive oil, the dose given being $1 \mathrm{drachm}$ of the mixture for every year of age. If anything an excess was given, and if the symptoms were severe and the enema well retained 1 or even 2 extra drachms were given. Before administering the enema the skin round the anus was well smeared with vaseline and the catheter then passed up 3 to 6 inches. The ether mixture was passed into the funnel and allowed to flow into the rectum by its own weight. The rate of flow can be watched through the glass tube connecting the catheter to the tube. This also serves as a gauge to see whether there is anything left in the tube. When all the enema has been run in, the catheter is rapidly withdrawn and usually none leaks out. Ether appears in the breath about five minutes after the enema has been given and can be detected during the next twenty-four hours. It has never in this series caused any digestive upset or pulmonary trouble. In many cases it did not begin to have any beneficial effect till after the second administration, and in severe cases actually aggravated the symptoms at first.

I treated the first 25 cases with ether alone without drugn and the next 25 with ether and drugs; the latter improved so much more rapidly than those treated with ether alone that the remainder of the cases were always treated with both. Of drugs I tried phenazone, bromides, belladonna, iodine, and opium in various combinations. I met with successful results with all these drugs, but belladonna was the most generally successful. In the cases summarized in this article only four enemas or injections were given-in some cases one each day and in others on alternate days. There would seem to be no advantage in resting for forty-eight hours nor any need to limit the treatment to four doses.

After December 1st, 1924, all cases of whooping-cough coming to the casualty department were treated by ether. In this way the results of 86 consecutive cases can be recorded. Of these, 28 were treated by injection and 58 by enema. Of the former, 11 gave up the treatment before the course was completed, but, with the exception of 3 , the results as far as they went were satisfactory. Three cases must be classed as failures, and the remaining 14 were successful.

Of those treated by enema 14 gave up the treatment owing to the parents' objections or because the child would not allow the administration of the enema. Of the remainder, 13 were failures and 31 successful. Of the children successfully treated, the earliest had been coughing for four days and the longest for more than four weeks. This case was a girl of 6 who had been coughing for about a month when first seen. She was having about forty attacks during the twenty-four hours, and vomited in nearly half of them. The appetite was very poor, and there were frequent attacks of epistaxis. The first two enemas contained 3 drachms of ether each, and the second two $3 \frac{1}{2}$ drachms. The breathing improved after the second, and the whooping completely stopped after the fourth. One week later the vomiting also stopped and the appetite improved. The child was putting on weight at the rate of an ounce a week, and reported satisfactory progress at every subsequent visit. No more epistaxis occurred after treatment was started.

In addition to these cases of pertussis treated in the casualty department, an outbreak among the in-patients enabled me to try the ether treatment on a number of cases under constant observation. Of these, 12 were treated by enemas and 5 by injections. Nine of the former were successful; the paroxysms were at once considerably diminished in number and violence, and ceased within a few days. Three were failures. Two of these patients wore already under treatment for bronchopneumonia and the third for anterior poliomyelitis. The observations of the nursing staff bear out what the mothers reported to me in the out-patient department. Of those treated by injection 4 were successful and 1 a failure, though benefited.

I have had no success with ether as a prophylactic, though probably, if given when a suspicious cough commences, the disease will be aborted or rendered rery mild. It is perhaps worth noting that the only cases in the ward which remained unaffected and were not given any prophylactic treatment were two cases of coeliac disease. They were in the ward when the original case was admitted, and at the time of writing, three months after the first "whoop" was heard, have no signs of the disease. 